\title{
Decreased activity of plasma ADAMTS-13 predicts poor prognosis in acute lymphoblastic leukemia patients after hematopoietic stem cell transplant
}

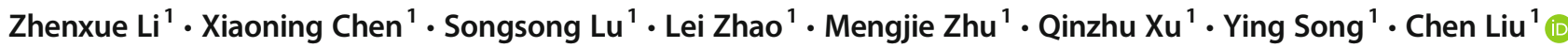

Received: 14 October 2019 / Accepted: 20 December 2019/Published online: 3 January 2020

(C) Springer-Verlag GmbH Germany, part of Springer Nature 2020

\begin{abstract}
Decreased ADAMTS-13 is important for the diagnosis of thrombotic thrombocytopenic purpura (TTP), but recent studies have shown that reduced ADAMTS-13 activity is associated with disease prognosis of a variety of cancer patients. As an important hematological malignancy, the significance of ADAMTS-13 in acute lymphoblastic leukemia (ALL) has not been fully elucidated. This research aims to explore the significance of decreased ADAMTS-13 activity in acute lymphoblastic leukemia after hematopoietic stem cell transplant (HSCT). Thirty-eight ALL patients were included in this research, and their ADAMTS-13 activity was measured before HSCT, including 21 patients with low ADAMTS- 13 activity $(<481 \mathrm{ng} / \mathrm{ml})$ and 17 patients with normal ADAMTS-13 activity (481-785 ng/ml). Related medical indicators before HSCT and 1 month after HSCT were collected. All the patients were followed and their progress was recorded and evaluated. Level changes of related medical indicators and prognosis situations were compared between two groups. ALL patients with low ADAMTS-13 activity suffered more HSCTrelated complications than patients with normal ADAMTS-13 activity. There was no significant difference for APTT, PT, CRP, and D-dimer between two groups. Low ADAMTS-13 Group underwent higher mortality rate than Normal Group during the 1year follow-up after HSCT and 2-year follow-up after the onset of ALL. To conclude, low ADAMTS-13 activity predicts poor prognosis of ALL after HSCT.
\end{abstract}

Keywords ADAMTS-13 $\cdot$ Acute lymphoblastic leukemia $\cdot$ HSCT $\cdot$ Prognosis

\section{Introduction}

As a common type of leukemia, acute lymphoblastic leukemia (ALL) is a serious hazard to human health. ALL includes leukemia of the $\mathrm{T}$ and $\mathrm{B}$ lineages and is characterized by overproduction and accumulation of lymphoblasts and could threaten individual life [1]. Hematopoietic stem cell transplantation (HSCT) is one of the important treatments for ALL patients. If the same sibling donor of HLA is available, hematopoietic stem cell transplantation is a good treatment option for many ALL patients [2]. However, HSCT also faces various threats, such as the main complications after HSCT, including graft-versus-host disease (GVHD), various infections, etc. [2].

Chen Liu

liuchen-best@pku.edu.cn

1 Department of Clinical Laboratory, Peking University People's Hospital, 11\# Xizhimen South Street, Beijing 100044, China
It is of great clinical and theoretical value to explore the factors affecting prognosis of ALL patients in the process of hematopoietic stem cell transplantation.

Von Willebrand factor (VWF) is a circulating glycoprotein in the blood. Its main functions include mediating the adhesion of platelets to damaged blood vessels [3, 4]. Mature VWF is a multimeric protein that is cleaved by a metalloproteinase of the ADAMTS family called ADAMTS-13 (a disintegrin and metalloprotease with thrombospondin motifs-13, also called von Willebrand factor-cleaving protease) [5]. ADAMTS-13 plays a crucial role in maintaining normal size of VWF multimers and is an important factor for VWF to function normally. Large VWF multimers accumulate under conditions of low levels of ADAMTS-13. A large increase of the concentration of VWF multimers can lead to excessive platelet agglutination, which could induce coagulation disorders $[6,7]$. Previous studies have reported that ADAMTS-13 activity less than $5 \%$ of normal plasma activity is an important cause of thrombotic thrombocytopenic purpura (TTP) [6-8]. 
The reduction in ADAMTS-13 activity is not only in TTP but also in a variety of metastatic malignancies or after surgery $[9,10]$. A significant thrombogenic phenotype has been reported in mice that are completely deficient in ADAMTS-13 $[11,12]$. Patients with acute systemic inflammation are with significantly reduced ADAMTS-13 activity [13], suggesting a possible association between inflammation and ADAMTS-13 deficiency. Previous studies found a decrease in ADAMTS-13 levels in ALL, which were significantly associated with inflammatory factors and risk stratification in ALL patients [14]. However, the importance of ADAMTS-13 during HSCT in ALL patients is still unclear.

In this study, we aimed to investigate the significance of the decrease of plasma ADAMTS-13 activity in ALL patients after HSCT and to initially assess the relationship between low ADAMTS-13 activity and prognosis in ALL patients after HSCT.

\section{Materials and methods}

\section{Patients}

According to the classification criteria for acute leukemia [15], 38 ALL patients who were admitted to the hospital from September 2017 to January 2018 and underwent a haploidentical allo-HSCT were collected. Their induction chemotherapy regimens included VDCLP (vincristine, daunorubicin, cyclophosphamide, L-asparaginase, and prednisone), VDCP, VDLP, or VDP, for one to two cycles. Conditioning regimen for allo-HSCT includes arabinoside, busulfan, cyclophosphamide, and semustine, along with ATG. Prophylactics for GVHD include cyclosporine (CsA), mycophenolate mofetil, and short-term methotrexate (MTX). The plasma ADAMTS-13 activity was measured before conditioning regimen of HSCT, and their medical records were followed up and collected. All of these patients had no kidney diseases, autoimmune diseases, other tumors, liver diseases, cardiovascular diseases, diabetes, DIC, drug intake, or thromboembolic events prior to HSCT. Patients with TTP were also excluded. The study was conducted in accordance with the ethical standards of the Helsinki Declaration, and all selected individuals have given informed consent. The study was approved by the ethics committee of the hospital.

\section{Blood sampling and analysis of ADAMTS-13 activity}

This study used the recombinant VWF86-ALEXA FRET substrate method to determine citrate anti-coagulated plasma samples to measure the activity of ADAMTS-13. Plasma ADAMTS-13 activity was determined using the ACTIFLUOR ADAMTS 13 Activity Assay Kit purchased from SEKISUI Diagnostics, LLC (Stamford, CT). A standard curve was constructed using normal plasma of known concentrations of ADAMTS-13. The activity of ADAMTS-13 in plasma was determined by measuring the sample and using the fluorescence change of the standard curve, and the activity was expressed as $\mathrm{ng} / \mathrm{ml}$ according to the curve. The local reference range (above 2.5 percentile and below 97.5 percentile) of ADAMTS-13 for this method was determined to be 481-785 ng/ml, and ADAMTS-13 less than $481 \mathrm{ng} / \mathrm{ml}$ was considered to be clinical low level.

\section{Clinical indicator measurement}

VWF, PT, APTT, and D-dimer levels were determined on ACL-TOP 700 multiparameter hemostasis analyzer (Instrumentation Laboratory Company, Bedford, MA, USA). CBC counts were measured by SYSMEX XE-2100 hematology analyzer (TOA Medical Electronics, Kobe, Japan). CRP was detected using i-CHROMA (Boditech Med Inc., Chuncheon, Korea).

\section{Statistics}

All analyses were performed using GraphPad Prime 5.5 software. All data are expressed as mean \pm SD unless otherwise stated. Student's t-test and $\chi 2$ statistics (Fisher's exact test) were used to assess differences between groups. Survival rates were analyzed using the Kaplan-Meier method and differences between groups were compared using the Mantel-Cox and Breslow tests. All statistical tests are two tails. A $P$ value less than 0.05 was considered to be statistically significant.

\section{Results}

\section{Clinical indicators of ALL patients measured before and after HSCT}

Totally 38 ALL patients admitted to the hospital who afterward underwent a haploidentical allo-HSCT were enrolled. ADAMTS-13 activity of the patients was measured before conditioning regimen of HSCT. According to the reference range of Chinese people (above 2.5 percentile and above 97.5 percentile), we divided the patients into two groups: Low ADAMTS-13 Group $(<481 \mathrm{ng} / \mathrm{ml})$ and Normal Group (481-785 ng/ml). All patients were without irrelevant diseases (including kidney diseases, autoimmune diseases, other tumors, liver diseases, cardiovascular diseases, diabetes, DIC, etc.) and TTP was excluded as well, according to the diagnosis of clinicians. The demographic and clinical characteristics of the ALL patients before conditioning regimen were listed in Table 1. There was no significant difference about the clinical characteristics (age, gender, and BMI) and the detailed constitutions ( $\mathrm{T}$ or B lineage) between two groups. The 
Table 1 Summary of the demographic and clinical characteristics of the ALL patients before HSCT

\begin{tabular}{llll}
\hline & Low $(n=21)$ & Normal $(n=17)$ & $P$ values \\
\hline Characteristics & & & \\
ADAMTS-13 (ng/ml) & $377.0 \pm 64.7$ & $623.8 \pm 119.3$ & 0.00 \\
& $<481$ & $\geq 481$ & \\
Gender (M/F) & $12 \mathrm{M} / 9 \mathrm{~F}$ & $8 \mathrm{M} / 9 \mathrm{~F}$ & 0.74 \\
Age (year) & $28.4 \pm 14.1$ & $29.8 \pm 10.8$ & 0.74 \\
& $14-58$ & $10-54$ & \\
BMI & $19.7 \pm 6.5$ & $20.2 \pm 7.5$ & 0.92 \\
Days from diagnosis & $275.0 \pm 94.7$ & $274.5 \pm 210.3$ & 0.99 \\
to BMT & & & \\
Hematologic parameter & & & \\
vWF (\%) & $117.9 \pm 32.1$ & $103.4 \pm 17.2$ & 0.08 \\
APTT(s) & $30.9 \pm 5.0$ & $30.5 \pm 4.7$ & 0.87 \\
PT(s) & $12.4 \pm 1.9$ & $12.4 \pm 1.7$ & 1.0 \\
Hgb (g/L) & $93.1 \pm 18.2$ & $90.2 \pm 15.4$ & 0.61 \\
PLT (109/L) & $64.8 \pm 13.7$ & $63.8 \pm 13.6$ & 0.85 \\
D-Dimer (ng/ml) & $931 \pm 1380$ & $956 \pm 1357$ & 0.95 \\
WBC (109/L) & $4.2 \pm 2.1$ & $3.7 \pm 2.2$ & 0.53 \\
CRP (mg/L) & $17.8 \pm 13.8$ & $15.4 \pm 19.5$ & 0.20 \\
Constitute & & & 1.0 \\
T lineage & 6 & 4 & \\
B lineage & 15 & 13 & \\
\hline
\end{tabular}

Data are present as mean $\pm \mathrm{SD}$; ALL, acute lymphoblastic leukemia; APTT, activated partial thromboplastin time; BMI, body mass index; HSCT, bone morrow transplant; CRP, C-reactive protein; PT, prothrombin time; vWF, von Willebrand factor; WBC, white blood cells

cytogenetics, white blood count at diagnosis, and HLA matching results were also analyzed, and there was no significant difference between groups as well (data not shown). We also calculated the time interval from diagnosis to BMT in the two groups of patients, $275.0 \pm 94.7$ days and $274.5 \pm$ 210.3 days, respectively, and there was no significant difference between the two groups (Table 1). Their follow-up medical records were collected. We evaluated APTT, PT, CRP, WBC, and hemoglobin values 1 month after HSCT, since the body's state has reached a relatively stable level at that time. The data are listed in Table 2. There was no significant difference for PT, APTT, CRP, and D-dimer between the two groups. We also measured vWF activity. However, the range of the values varied considerably, and there was no significant difference between groups.

\section{ALL patients in Low ADAMTS-13 Group suffered more HSCT-related complications than patients in Normal Group}

All the patients were followed up for 1 year after HSCT. HSCT-related complications of each patient was recorded,
Table 2 Summary of the clinical characteristics of the ALL patients 1 month after HSCT

\begin{tabular}{|c|c|c|c|}
\hline & Low $(n=21)$ & Normal $(n=17)$ & $P$ values \\
\hline ADAMTS-13(ng/ml) & $321.5 \pm 78.5$ & $687.6 \pm 162.5$ & 0.00 \\
\hline \multicolumn{4}{|l|}{ Hematologic parameter } \\
\hline $\mathrm{vWF}(\%)$ & $128.5 \pm 45.2$ & $97.4 \pm 31.9$ & 0.76 \\
\hline APTT(s) & $32.3 \pm 6.9$ & $35.5 \pm 10.5$ & 0.29 \\
\hline $\mathrm{PT}(\mathrm{s})$ & $12.2 \pm 2.0$ & $14.2 \pm 6.4$ & 0.23 \\
\hline $\operatorname{Hgb}(g / L)$ & $81.4 \pm 17.8$ & $81.5 \pm 17.5$ & 0.99 \\
\hline PLT(109/L) & $52.2 \pm 53.9$ & $49.7 \pm 51.0$ & 0.88 \\
\hline D-Dimer(ng/ml) & $1233 \pm 2149$ & $2916 \pm 5167$ & 0.22 \\
\hline WBC(109/L) & $4.4 \pm 3.0$ & $3.7 \pm 2.6$ & 0.46 \\
\hline $\mathrm{CRP}(\mathrm{mg} / \mathrm{L})$ & $13.4 \pm 21.5$ & $28.0 \pm 47.7$ & 0.25 \\
\hline \multicolumn{4}{|l|}{ Complications } \\
\hline Pulmonary infection & $14(67 \%)$ & $5(29 \%)$ & 0.049 \\
\hline GVHD & $13(62 \%)$ & $11(65 \%)$ & 1.0 \\
\hline GIB & $4(19 \%)$ & $2(12 \%)$ & 0.67 \\
\hline $\mathrm{ARF}$ & $5(24 \%)$ & $2(12 \%)$ & 0.43 \\
\hline CMV infection & $13(62 \%)$ & $4(24 \%)$ & 0.02 \\
\hline TMA & $3(14 \%)$ & $1(6 \%)$ & 0.61 \\
\hline Respiratory failure & $6(29 \%)$ & $2(12 \%)$ & 0.26 \\
\hline Hypoproteinemia & $7(33 \%)$ & $2(12 \%)$ & 0.15 \\
\hline EBV infection & $3(14 \%)$ & $1(6 \%)$ & 0.61 \\
\hline Hepatic injury & $3(14 \%)$ & $1(6 \%)$ & 0.61 \\
\hline
\end{tabular}

Data are present as mean \pm SD for clinical characteristics; ALL, acute lymphoblastic leukemia; APTT, activated partial thromboplastin time; ARF, acute renal failure; HSCT, bone morrow transplant; CRP, Creactive protein; GIB, gastrointestinal bleeding; GVHD, graft-versushost disease; PT, prothrombin time; TMA, thrombotic microangiopathies; vWF, von Willebrand factor; WBC, white blood cells

including infection, bleeding, GVHD, organ failure, and thrombotic microangiopathies (TMA). Numbers of occurrence in two groups were listed in Table 2. We found that the percentage of each complication was higher in Low ADAMTS-13 Group than that in Normal Group. For pulmonary infection and cytomegalovirus infection, Low ADAMTS-13 Group suffered significant higher percentage of occurrence than Normal Group.

\section{Low ADAMTS-13 Group has higher mortality rate than Normal Group}

All patients were followed for 1 year and the situations of mortality were recorded. The deaths were caused by nonrelapse mortality (e.g., infections or GVHD) or relapse that was not curable, or mixed reason. The Kaplan-Meier method was used to analyze survival, and the two groups were compared by use of the Mantel-Cox and Breslow tests. The survival curve is shown in Fig. 1, Mantel-Cox test between curves, $P=0.0290$; Breslow test between curves, $P=0.0385$. 


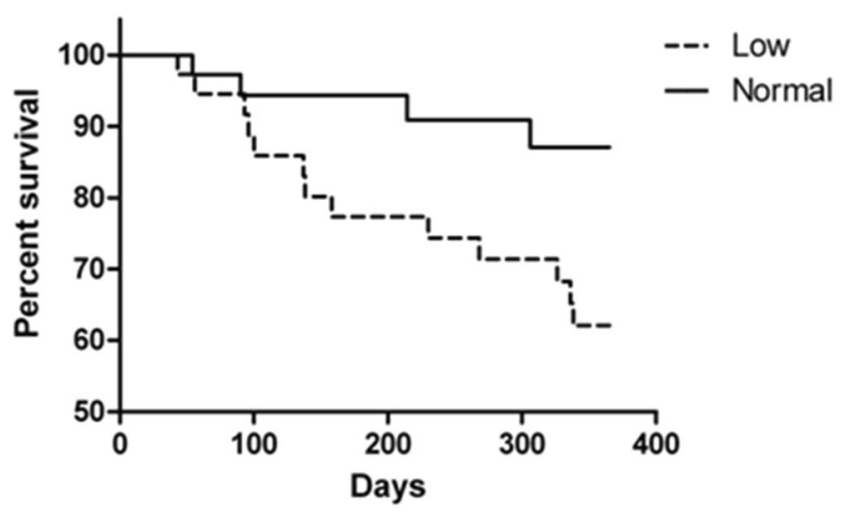

Mantel-Cox Test $\mathrm{P}=0.0290$

\section{Gehan-Breslow-Wilcoxon Test $\mathrm{P}=0.0385$}

Fig. 1 ALL patients were followed for 1 year after HSCT, and the Kaplan-Meier curves were used to show percentage of survival of Low ADAMTS-13 Group (dashed) and Normal Group (solid). Two groups were compared by use of the Mantel-Cox and Breslow tests and the $P$ values were listed below

We also analyze the survival curve started from onset of ALL. Situation of each patient was recorded for 2 years (24 months) since onset of ALL, which could exclude the influence of the time course from onset to HSCT. As shown in Fig. 2, Mantel-Cox test between curves, $P=0.0115$, and Breslow test between curves, $P=0.0186$, and there was still significant difference between two groups.

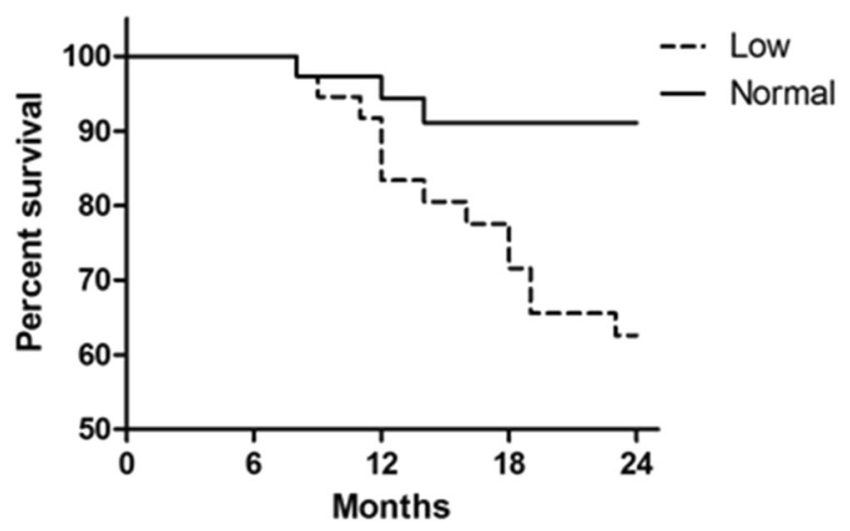

Mantel-Cox Test $\mathrm{P}=0.0115$

\section{Gehan-Breslow-Wilcoxon Test $\mathrm{P}=0.0186$}

Fig. 2 The survival curve started from the day of onset, followed for 24 months since the day the leukemia was onset. The Kaplan-Meier curves were used to show percentage of survival of Low ADAMTS-13 Group (dashed) and Normal Group (solid) of ALL patients. $P$ values of the Mantel-Cox and Breslow tests were listed below

\section{Discussion}

The change in activity of ADAMTS-13 has an important effect on the body's coagulation function. Low levels of ADAMTS-13 activity were considered to be the cause of TTP and had been thoroughly studied and used to diagnose TTP [5-7]. Although not as low as in TTP, the significance of the reduction in ADAMTS-13 activity in leukemia still needs to be given sufficient attention. Previous study showed a decrease in the activity of ADAMTS-13 in both ALL and AML patients, which was associated with inflammatory factors and risk stratifications $[14,16]$. Low ADAMTS-13 activity was also a prognostic risk factor for AML [16]. In addition, patients with low ADAMTS13 activity had a worse prognosis and had more serious complications in AML patients who underwent hematopoietic stem cell transplantation [17]. In this study, we also demonstrated that ADAMTS-13 activity levels can reflect the prognosis of ALL patients after HSCT. For patients with ADAMTS-13 activity below $481 \mathrm{ng} / \mathrm{ml}$, the probability of serious complications and mortality was higher than that in patients with normal ADAMTS-13 activity, suggesting that ADAMTS-13 has a potential predictive effect on the prognosis of ALL after HSCT.

To answer how ADAMTS-13 could reduce the rate of survival, it is necessary to expand the sample size for intensive research. Infection and recurrence may all be influencing factors (we also tested the recurrence rate of the two groups but did not reach a statistically significant level). We believe that the reduction of the effect of ADAMTS-13 on coagulation is also a potential factor, which may be associated with changes in coagulation function in patients. Although the level of ADAMTS-13 does not reach a level as low as TTP patients to cause severe clinical symptoms, the decreased level of ADAMTS-13 may also be meaningful in the progress of ALL during HSCT. Because of the higher risk of excessive platelet aggregation, coagulation may get worse, and further it could lead to organ failure and even death.

ADAMTS-13 activity is reduced in patients with acute systemic inflammation [13, 14], so the local inflammatory response of ALL patients before and after HSCT may play an important role. But according to our results, CRP levels did not differ significantly between two groups. Lasparaginase is known to alter many coagulation and anticoagulant proteins, whereas ALL patients are often treated with L-asparaginase in chemotherapy [18, 19], and L-asparaginase may be a potential cause of ADAMTS-13 activity, which is worth researching in detail in the future. Lower translational activity associated with the ADAMTS-13 rs2285489 C/C genotype in hepatic stellate cells might contribute to an increased incidence of relapse [20], which suggests that ADAMTS-13 
single-nucleotide polymorphism may also contribute to the role of ADAMTS-13 in prognosis of HSCT for leukemia patients. In addition, it should be mentioned that consumptive coagulopathy and liver dysfunction are also important factors for the reduction of ADAMTS-13, although we had excluded patients with DIC and liver disease in this study.

We found that VWF activity was inconsistent with changes in ADAMTS-13 activity and there was no significant difference in vWF levels between the two groups before HSCT, although ADAMTS-13 activity was significantly different between the two groups. This may be due to insufficient reduction in ADAMTS-13 activity to severely affect $\mathrm{vWF}$ activity.

This study still has many limitations. The number of ALL patients is not very large. In addition, mechanisms about reduction of ADAMTS-13 and how ADAMTS-13 affect prognosis after HSCT are still unclear and need to be studied in the future. To conclude, we explored the role of low ADAMTS13 activity in the prognosis of ALL patients after HSCT, which will help to fully understand the clinical significance of ADAMTS-13 in leukemia, especially its predictive effect on the prognosis of acute leukemia.

Acknowledgments We thank hematology department of Perking University People's Hospital for the sharing of medical records.

This manuscript abstract was partly presented as a poster for the 30th World Congress of World Association of Societies Pathology and Laboratory Medicine (WASP\&LM2019) at Xi'an, China, on Sept 1719, 2019.

Authors' contributions CL took charge of all the work and participated in its design and carried out part of the experiments. ZL carried out part of the experiments and modified the manuscript. XC carried out part of the experiments. SL and LZ drafted the manuscript. MZ and QX did part of the clinical measurements. YS helped to modify the manuscript and contributed to the concept as well.

All authors read and approved the final manuscript.

Funding information This work was supported by grants from Natural Science Foundation of China $(81871230,81401298)$ and Beijing Natural Science Foundation (7163228).

\section{Compliance with ethical standards}

Competing interests The authors state that there is no conflict of interest.

\section{References}

1. Pui, C H (2011) Acute lymphoblastic leukemia (pp. 23-26). Springer Berlin Heidelberg

2. Bassan R, Hoelzer D (2011) Modern therapy of acute lymphoblastic leukemia. J Cin Oncol 29:532-543. https://doi.org/10.1200/ JCO.2010.30.1382
3. Bryckaert M, Rosa JP, Denis CV, Lenting PJ (2015) Of von Willebrand factor and platelets. Cell Mol Life Sci 72:307-326. https://doi.org/10.1007/s00018-014-1743-8

4. Lenting PJ, Christophe OD, Denis CV (2015) von Willebrand factor biosynthesis, secretion, and clearance: connecting the far ends. Blood 125:2019-2028. https://doi.org/10.1182/blood-2014-06528406

5. Zheng X, Chung D, Takayama TK, Majerus EM, Sadler JE, Fujikawa K (2001) Structure of von Willebrand factor-cleaving protease (ADAMTS-13), a metalloprotease involved in thrombotic thrombocytopenic purpura. J Biol Chem 276:41059-41063. https:// doi.org/10.1074/jbc.C100515200

6. Zheng XL (2015) ADAMTS-13 and von Willebrand factor in thrombotic thrombocytopenic purpura. Annu Rev Med 66:211225. https://doi.org/10.1146/annurev-med-061813-013241

7. Bianchi V, Robles R, Alberio L, Furlan M, Lämmle B (2002) Von Willebrand factor-cleaving protease (ADAMTS-13) in thrombocytopenic disorders: a severely deficient activity is specific for thrombotic thrombocytopenic purpura. Blood 100:710-713. https://doi. org/10.1182/blood-2002-02-0344

8. Sadler JE (2008) Von Willebrand factor, ADAMTS-13, and thrombotic thrombocytopenic purpura. Blood 112:11-18. https://doi.org/ 10.1182/blood-2008-02-078170

9. Oleksowicz L, Bhagwati N, DeLeon-Fernandez M (1999) Deficient activity of von Willebrand's factor-cleaving protease in patients with disseminated malignancies. Cancer Res 59:22442250

10. Pereboom ITA, Adelmeijer J, Van Leeuwen Y, Hendriks HGD, Porte RJ, Lisman T (2009) Development of a severe von Willebrand factor/ADAMTS-13 dysbalance during orthotopic liver transplantation. Am J Transplant 9:1189-1196. https://doi.org/10. 1111/j.1600-6143.2009.02621.x

11. Banno F, Kokame K, Okuda T, Honda S, Miyata S, Kato H, Tomiyama Y (2006) Complete deficiency in ADAMTS-13 is prothrombotic, but it alone is not sufficient to cause thrombotic thrombocytopenic purpura. Blood 107:3161-3166. https://doi.org/ 10.1182/blood-2005-07-2765

12. Chauhan AK, Motto DG, Lamb CB, Bergmeier W, Dockal M, Plaimauer B, Scheiflinger F, Ginsburg D, Wagner D et al (2006) Systemic antithrombotic effects of ADAMTS-13. J Exp Med 203: 767-776. https://doi.org/10.1084/jem.20051732

13. Reiter RA, Varadi K, Turecek PL, Jilma B, Knöbl P (2005) Changes in ADAMTS-13 (von-Willebrand-factor-cleaving protease) activity after induced release of von Willebrand factor during acute systemic inflammation. Thromb Haemost 93:554-558. https://doi.org/10. 1160/TH04-08-0467

14. Liu C, Zhao L, Zhao J, Xu Q, Song Y, Wang H (2017) Decreased ADAMTS-13 level is related to inflammation factors and risk stratification of acute lymphoblastic leukemia patients. Medicine 96: e6136. https://doi.org/10.1097/MD.0000000000006136

15. Bennett JM, Catovsky D, Daniel MT, Flandrin G, Galton DA, Gralnick HR, Sultan C (1976) Proposals for the classification of the acute Leukaemias French-American-British (FAB) co-operative group. Brit J Haematol 33:451-458. https://doi.org/10.1111/j.13652141.1976.tb03563.x

16. Liu C, Zhao L, Zhao J, Xu Q, Song Y, Wang H (2017) Reduced ADAMTS-13 level negatively correlates with inflammation factors in plasma of acute myeloid leukemia patients. Leuk Res 53:57-64. https://doi.org/10.1016/j.leukres.2016.12.004

17. Liu C, Han M, Zhao L, Zhu M, Xu Q, Song Y, Wang H (2019) ADAMTS-13 activity reduction in plasma of acute myeloid leukemia predicts poor prognosis after hematopoietic stem cell transplantation. Hematology 24:129-133. https://doi.org/10.1080/ 10245332.2018.1532648

18. Truelove E, Fielding AK, Hunt BJ (2013) The coagulopathy and thrombotic risk associated with L-asparaginase treatment in adults 
with acute lymphoblastic leukaemia. Leukemia 27:553-559. https://doi.org/10.1038/leu.2012.290

19. Goyal G, Bhatt VR (2015) L-asparaginase and venous thromboembolism in acute lymphocytic leukemia. Future Oncol 11:24592470. https://doi.org/10.2217/fon. 15.114

20. Nomoto H, Takami A, Espinoza JL, Onizuka M, Kashiwase K, Morishima Y, Fukuda T, Kodera Y, Doki N, Miyamura K, Mori T, Nakao S, Morishita E (2019) Recipient ADAMTS-13 single- nucleotide polymorphism predicts relapse after unrelated hematopoietic stem cell transplantation for hematologic malignancy. Int $\mathrm{J}$ Mol Sci 20:214. https://doi.org/10.3390/ijms20010214

Publisher's note Springer Nature remains neutral with regard to jurisdictional claims in published maps and institutional affiliations. 